\title{
COMPOSTOS ORGANOLANTANÍDEOS CONTENDO O LIGANTE PIRAZINAMIDA: SÍNTESE, CARACTERIZAÇÃ̃O E ATIVIDADE CATALÍTICA NA POLIMERIZAÇÃo DO ETILENO
}

\author{
Renata Diana Miotti \\ Centro Universitário FIEO, UNIFIEO, Av. Franz Voegeli, 300, 06020-190 Osasco - SP \\ Alessandra de Souza Maia e Wanda de Oliveira* \\ Instituto de Química, Universidade de São Paulo, CP 26077, 05513-970 São Paulo - SP \\ Ícaro Sampaio Paulino e Ulf Schuchardt \\ Instituto de Química, Universidade Estadual de Campinas, CP 6154, 13083-970 Campinas - SP
}

Recebido em 4/9/01; aceito em 9/1/02

\begin{abstract}
ORGANOLANTHANIDE COMPOUNDS CONTAINING THE PIRAZINAMIDE LIGAND: SYNTHESIS, CHARACTERIZATION AND CATALYTIC ACTIVITY IN ETHYLENE POLYMERIZATION: In this work we report the synthesis of some organolanthanide compounds which were identified as $\mathrm{LnCl}_{2} \mathrm{Cp}(\mathrm{PzA})_{2}, \mathrm{Ln}=\mathrm{Nd}, \mathrm{Sm}, \mathrm{Eu}$ and $\mathrm{Tb}, \mathrm{Cp}=$ cyclopentadienyl and PzA = pirazinamide, by elemental analyses, complexometric titration with EDTA, thermal analyses and IR spectra. Thermal analysis and infrared spectra indicated that the coordination of the pyrazinamide to the lanthanide ions was made by the $\mathrm{O}$ atom of the carbonyl group and by one or both $\mathrm{N}$ atoms of the pyrazinamide ring. This class of compound showed catalytic activity of ca. 4.0 to $6.4 \mathrm{kgPE} \mathrm{molLn}^{-1} \mathrm{~h}^{-1} \mathrm{bar}^{-1}$, in ethylene polymerization, using methylaluminoxane as cocatalyst. The resulting polyethylene presented low crystallinity $(20 \%)$.
\end{abstract}

Keywords: organolanthanides; cyclopentadienyl; ethylene.

\section{INTRODUÇÃO}

Nós vivemos na idade do polímero. Plásticos, fibras, elastômeros, celulose, borracha, adesivos são todos termos comuns em nosso vocabulário moderno e todos são parte do fascinante mundo da química polimérica. A descoberta de processos catalíticos nesta área foi de fundamental importância para o desenvolvimento da ciência dos polímeros como, por exemplo, para a produção industrial de poliolefinas.

No princípio dos anos 50, rotas de síntese de polietileno de alta densidade, em condições brandas (baixa temperatura e pressão), foram desenvolvidas a partir dos trabalhos de Ziegler e Natta, utilizando-se como catalisadores haletos de metais de transição combinados com alquil-alumínio ${ }^{1}$. Como resultado destes trabalhos, foram estudados vários sistemas catalíticos, um deles baseado em compostos metalocênicos e metilaluminoxano que apresenta atividade catalítica bastante elevada em polimerização de etileno em condições bran$\operatorname{das}^{2}$.

Nas últimas duas décadas uma química extensa se desenvolveu então no campo dos compostos organolantanídeos que continham o ligante ciclopentadienil ou alguns de seus derivados, devido às suas aplicações na polimerização de olefinas, em particular do etileno ${ }^{3}$.

Considerando os avanços no campo de novos catalisadores para polimerização de olefinas, um trabalho voltado para a aplicação de organolantanídeos como catalisadores na polimerização de etileno envolvendo o estudo da síntese de compostos organolantanídeos do tipo $\mathrm{LnCl}_{2} \mathrm{Cp}(\mathrm{PzA})_{2}, \mathrm{Ln}=\mathrm{Nd}, \mathrm{Sm}$, Eu e Tb, $\mathrm{Cp}=$ ciclopentadienil e $\mathrm{PzA}=$ pirazinamida é aqui apresentado. Análise elementar, termogravimetria e espectros na região do infravermelho foram utilizados para caracterizar estes compostos. A atividade catalítica de tais compostos foi investigada na polimerização do etileno na presença de metilaluminoxano.

*e-mail: wdolivei@quim.iq.usp.br
Os compostos organolantanídeos são normalmente obtidos ${ }^{4}$ pela reação entre os sais anidros de lantanídeos e o $\mathrm{NaCp}$, seguida da introdução dos ligantes. A utilização de sais anidros é essencial para a formação dos compostos organolantanídeos, uma vez que estes compostos são extremamente sensíveis à umidade. No presente trabalho, para se obter os compostos organolantanídeos $\mathrm{LnCl}_{2} \mathrm{Cp}(\mathrm{PzA})_{2}$, o reagente de partida contendo o íon lantanídeo(III) deveria ser o cloreto de lantanídeo anidro, porém um dos problemas que ocorre ao se trabalhar especialmente com sais de lantanídeos anidros é a dificuldade em desidratá-los sem que haja a decomposição com formação de oxicloretos, devendo-se então controlar rigorosamente a sua desidratação. Uma maneira de se contornar esta dificuldade é se utilizar como reagente de partida não sais anidros, mas compostos de coordenação anidros contendo os ligantes desejados. Assim, neste trabalho utilizou-se uma rota alternativa para se obter os derivados organolantanídeos, partindo-se de compostos de coordenação anidros contendo o ligante desejado e reagindo-os com o ciclopentadienil de sódio.

\section{PARTE EXPERIMENTAL}

\section{Geral}

Todas as manipulações foram feitas sob argônio pré-purificado. Os solventes foram purificados e secos através de técnicas padrões ${ }^{5}$ e completamente desoxigenados antes do uso.

As micro-análises $(\% \mathrm{C}, \% \mathrm{H}, \% \mathrm{~N})$ foram feitas em um analisador Perkin-Elmer CHN 2400. A \%Ln foi determinada através de titulação complexométrica com $\mathrm{EDTA}^{6}$. As curvas termogravimétricas foram registradas em um analisador Shimadzu Thermogravimetric -TGA50, aquecendo-se 2 a $5 \mathrm{mg}$ de amostra em cadinho de platina, da temperatura ambiente a $800{ }^{\circ} \mathrm{C}$, a uma taxa de aquecimento de $20^{\circ} \mathrm{C} / \mathrm{min}$ em ar sintético $(50,0 \mathrm{~mL} / \mathrm{min})$. Os espectros na região do infravermelho foram registrados em um espectrômetro FTIRBOMEM; modelo MB-102, de 4000 a $200 \mathrm{~cm}^{-1}$, usando-se disper- 
sões dos compostos em Nujol ou em Fluorolube, entre janelas de iodeto de césio. As curvas DSC (Calorimetria diferencial exploratória) dos polímeros foram medidas em um DSC 2910 - TA Instruments. As amostras foram aquecidas de 25 a $200{ }^{\circ} \mathrm{C}$ a uma taxa de aquecimento de $10{ }^{\circ} \mathrm{C} / \mathrm{min}$. A temperatura de fusão $\left(\mathrm{T}_{\mathrm{f}}\right)$ e o calor de fusão $\left(\mathrm{DH}_{\mathrm{f}}\right)$ foram estimados a partir da segunda curva de aquecimento. $\mathrm{O}$ grau de cristalinidade foi calculado a partir do $\Delta \mathrm{H}_{\mathrm{f}}$, usando - se a equação $^{7}: \lambda_{\Delta C S}=\Delta \mathrm{H}_{\mathrm{f}} \times 100 / 288$.

\section{Síntese de $\mathrm{LnCl}_{2} \mathrm{Cp}(\mathrm{PzA})_{2}$}

Os compostos foram obtidos pela reação entre $\mathrm{NaCp}$ e $\mathrm{LnCl}_{3}(\mathrm{PzA})_{4}$ em THF, conforme método desenvolvido em nosso laboratório $^{8}$, obedecendo-se a proporção molar de $3 \mathrm{NaCp}$ : $1 \mathrm{LnCl}_{3}(\mathrm{PzA})_{4}, \mathrm{Ln}=\mathrm{Nd}, \mathrm{Sm}$, Eu e Tb. Os compostos de coordenação $\mathrm{LnCl}_{3}(\mathrm{PzA})_{4}$, foram obtidos e caracterizados segundo procedimentos descritos por Miotti ${ }^{9}$. Estes compostos de coordenação foram adicionados à solução de $\mathrm{NaCp}$ em THF e a mistura foi mantida sob agitação constante por aproximadamente $24 \mathrm{~h}$. Fez-se então a retirada da porção sobrenadante, com auxílio de uma seringa e, em seguida, a lavagem do resíduo com etanol anidro. O sólido assim obtido foi seco a vácuo à temperatura ambiente por cerca de $8 \mathrm{~h}$ e armazenado em atmosfera inerte. Rendimento do processo $70 \%$.

\section{Polimerização catalítica de etileno}

A polimerização foi feita em um reator Büchi de $1 \mathrm{~L}$, a $70{ }^{\circ} \mathrm{C}$ e 3 bar de etileno, usando $2,0 \mathrm{mg}$ do composto organolantanídeo e $2,0-2,5 \mathrm{~mL}$ de metilaluminoxano $(10 \%$ em tolueno) em $50 \mathrm{~mL}$ de tolueno. A reação de polimerização foi interrompida, após duas horas, pela adição de etanol. O polímero obtido foi lavado com etanol e seco a $50{ }^{\circ} \mathrm{C}$ por $5 \mathrm{~h}$. Uma solução do polímero $(10 \mathrm{mg})$ em decalina $(10 \mathrm{~mL})$ foi preparada a $140{ }^{\circ} \mathrm{C}$ e evaporada formando um filme de polietileno (utilizado para registro do espectro na região do infravermelho).

\section{RESULTADOS E DISCUSSÃO}

Neste trabalho utilizou-se a rota alternativa para se obter os derivados organolantanídeos, onde o ciclopentanienil de sódio $(\mathrm{NaCp})$ reagiu com $\mathrm{LnCl}_{3}(\mathrm{PzA})_{4}$ em THF, a temperatura ambiente, formando os compostos $\mathrm{LnCpCl}_{2}(\mathrm{PzA})_{2}, \mathrm{Ln}=\mathrm{Nd}, \mathrm{Sm}$, Eu e Tb, assim formulados a partir dos resultados da análise elementar (Tabela 1). Esta alteração de rota eliminou uma etapa muito crítica que é a desidratação dos sais de partida, neste caso dos cloretos de lantanídeos hidratados e não comprometeu o rendimento do processo que ficou em torno de $70 \%$.

Estes compostos mostraram-se praticamente insolúveis, em acetona, acetonitrila, benzeno, clorofórmio, etanol, metanol, nitrometano e THF, não sendo possível se obter monocristal para caracterização por difração de raios-X. Para auxiliar a caracterização dos compostos organolantanídeos foram analisadas as curvas TG/DTG.

Os compostos $\mathrm{LnCl}_{2} \mathrm{Cp}(\mathrm{PzA})_{2}$, mostraram características de decomposição semelhantes nas análises termogravimétricas, perdendo massa gradualmente com o aumento da temperatura de ca. 30 a $800{ }^{\circ} \mathrm{C}$, formando-se, nesta temperatura, $\mathrm{LnOCl}$ (Tabela 2). Uma curva TG/DTG típica desses compostos está apresentada na Figura 1. A formulação do resíduo como $\mathrm{LnOCl}$, oxicloreto de lantanídeo(III),
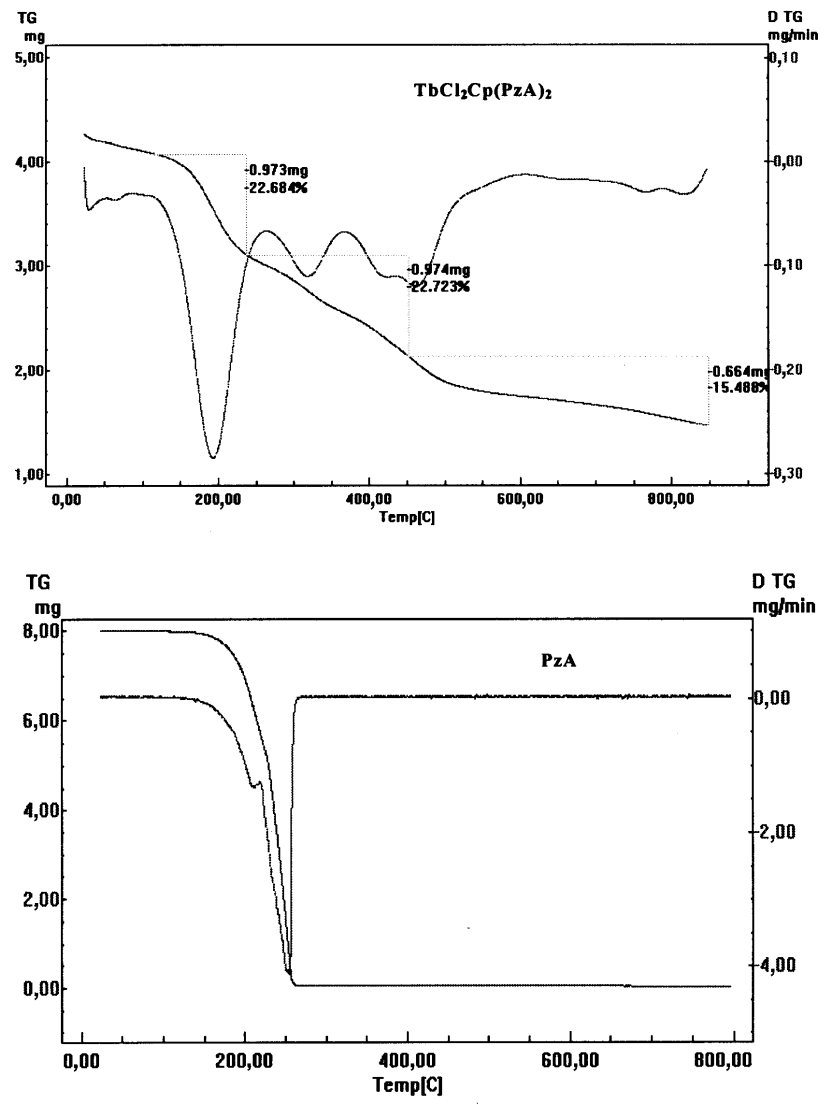

Figura 1. Curvas TG/DTG do composto $\mathrm{TbCl}_{2} \mathrm{Cp}(\mathrm{PzA})_{2}$ e da $\mathrm{PzA}$

Tabela 1. Resultados da análise elementar de $\mathrm{LnCl}_{2} \mathrm{Cp}(\mathrm{PzA})_{2}$

\begin{tabular}{lcccccccccc}
\hline composto & $\%$ Ln $_{\text {calc }}$ & $\% \mathrm{Ln}_{\text {exp }}$ & $\%_{\text {calc }}$ & $\%_{\text {exp }}$ & $\%_{\text {calc }}$ & $\%_{\text {exp }}$ & $\%_{\text {calc }}$ & $\%_{\text {exp }}$ & $\%_{\text {calc }}$ & $\%_{\text {exp }}$ \\
\hline $\mathrm{NdCl}_{2} \mathrm{Cp}(\mathrm{PzA})_{2}$ & 27,40 & 27,90 & 13,50 & 13,82 & 34,22 & 33,72 & 2,87 & 2,91 & 15,96 & 15,27 \\
$\mathrm{SmCl}_{2} \mathrm{Cp}(\mathrm{PzA})_{2}$ & 28,21 & 28,68 & 13,35 & 13,67 & 33,82 & 33,07 & 2,84 & 2,95 & 15,77 & 16,03 \\
$\mathrm{EuCl}_{2} \mathrm{Cp}(\mathrm{PzA})_{2}$ & 28,45 & 29,15 & 13,30 & 13,40 & 33,72 & 33,44 & 2,83 & 2,94 & 15,73 & 15,63 \\
$\mathrm{TbCl}_{2} \mathrm{Cp}(\mathrm{PzA})_{2}$ & 29,37 & 29,13 & 13,12 & 13,24 & 33,29 & 33,45 & 2,79 & 2,98 & 15,52 & 15,74 \\
\hline
\end{tabular}

Tabela 2. Termogravimetria de $\mathrm{LnCl}_{2} \mathrm{Cp}(\mathrm{PzA})_{2}$

\begin{tabular}{lcccccc}
\hline composto & \% perda total & m(inicial) $\mathrm{mg}$ & m(resíduo) $\mathrm{mg}$ & resíduo & MM calc g/mol & MM (TG) g/mol \\
\hline $\mathrm{NdCl}_{2} \mathrm{Cp}(\mathrm{PzA})_{2}$ & 63,31 & 1,231 & 0,452 & $\mathrm{NdOCl}$ & 526 & 534 \\
$\mathrm{SmCl}_{2} \mathrm{Cp}(\mathrm{PzA})_{2}$ & 61,74 & 3,511 & 1,344 & $\mathrm{SmOCl}$ & 532 & 527 \\
$\mathrm{EuCl}_{2} \mathrm{Cp}(\mathrm{PzA})_{2}$ & 62,05 & 3,810 & 1,446 & $\mathrm{EuOCl}$ & 534 & 536 \\
$\mathrm{TbCl}_{2} \mathrm{Cp}(\mathrm{PzA})_{2}$ & 60,89 & 4,288 & 1,677 & $\mathrm{TbOCl}$ & 541 & 537 \\
\hline
\end{tabular}


está de acordo com os estudos da decomposição térmica de alguns cloretos de lantanídeos hidratados ${ }^{9,10}$ e da constatação de que o ligante (PzA) não deixa resíduo nestas condições (Figura 1). A formulação do resíduo como $\mathrm{LnOCl}$ foi confirmada, também através do aquecimento estático das amostras realizado em atmosfera de ar, nas mesmas condições de aquecimento em que foram registradas as curvas termogravimétricas. No resíduo obtido pelo aquecimento estático foi feita a determinação da \%Ln por titulação complexométrica ${ }^{9}$.

A partir das variações de massas observadas nas curvas TG/DTG dos compostos $\mathrm{LnCl}_{2} \mathrm{Cp}(\mathrm{PzA})_{2}$, pode-se observar que a perda de massa ocorre em três etapas. A primeira etapa corresponde à perda de uma das duas moléculas de PzA e ocorre entre 25 e $250{ }^{\circ} \mathrm{C}$, devido à transformação de $\mathrm{LnCl}_{2} \mathrm{Cp}(\mathrm{PzA})_{2}$ para $\mathrm{LnCl}_{2} \mathrm{CpPzA}$. Entre $250 \mathrm{e}$ $400{ }^{\circ} \mathrm{C}$ tem-se a saída da molécula de PzA do composto $\mathrm{LnCl}_{2} \mathrm{CpPzA}$, formando $\mathrm{LnCl}_{2} \mathrm{Cp}$ e, finalmente entre 400 e $800^{\circ} \mathrm{C}$ tem-se a formação de $\mathrm{LnOCl}$ devido à decomposição de $\mathrm{LnCl}_{2} \mathrm{Cp}$ em atmosfera de ar. Na Tabela 3 estão apresentadas as variações de massas associadas a estas decomposições.

Os espectros na região do infravermelho dos compostos $\mathrm{LnCl}_{2} \mathrm{CpPzA}, \mathrm{Ln}=\mathrm{Nd}$, Sm, Eu e Tb (Tabela 4) mostraram um deslocamento da banda atribuída ao estiramento $\mathrm{C}=\mathrm{O}\left(\mathrm{v}_{\mathrm{CO}}\right) \mathrm{da}$ pirazinamida livre de $1680 \mathrm{~cm}^{-1}$ para $1642-1646 \mathrm{~cm}^{-1}$ nos compostos organolantanídeos, indicando a coordenação de uma molécula de pirazinamida ao íon lantanídeo(III) por meio do oxigênio do grupo de carbonila; e um deslocamento das bandas atribuídas à vibração do anel da pirazinamida livre de $1515 \mathrm{~cm}^{-1} ; 615 \mathrm{~cm}^{-1}$ e $435 \mathrm{~cm}^{-1}$ para $1617 \mathrm{~cm}^{-1} ; 664 \mathrm{~cm}^{-1}$ e $455 \mathrm{~cm}^{-1}$ nos compostos organolantanídeos, indicando que a coordenação da outra molécula de pirazinamida está ocorrendo via um ou ambos $\mathrm{N}$ do ane ${ }^{11-13}$. A atribuição dos modos vibracionais do ânion ciclopentadienil indicaram uma coordenação $\sigma$-centrada deste ao íon lantanídeo(III), com caráter iônico ${ }^{14}$.

Os espectros na região do infravermelho dos compostos organolantanídeos $\mathrm{LnCl}_{2} \mathrm{Cp}(\mathrm{PzA})_{2}, \mathrm{Ln}=\mathrm{Nd}, \mathrm{Sm}, \mathrm{Eu}, \mathrm{Tb}$, mostra- ram que as duas moléculas de PzA se coordenam de maneira diferente ao íon lantanídeo(III), uma pelo oxigênio do grupo carbonila da PzA e outra por um ou ambos $\mathrm{N}$ do anel da PzA, isto também foi evidenciado pelas curvas TG/DTG, onde temos a perda de cada molécula de pirazinamida em diferentes faixas de temperatura.

Os compostos $\mathrm{LnCl}_{2} \mathrm{Cp}(\mathrm{PzA})_{2}, \mathrm{Ln}=\mathrm{Nd}, \mathrm{Sm}$, Eu e Tb, apre-

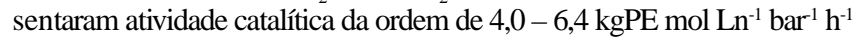
a $70{ }^{\circ} \mathrm{C}$ com relação Al/Ln igual a 2000 e 3 bar de pressão de etileno. Nesta série de compostos organolantanídeos observa-se apenas um pequeno aumento da atividade catalítica para o composto do íon $\mathrm{Tb}^{3+}$ (6,4 kg PE mol $\mathrm{Ln}^{-1}$ bar $\left.^{-1} \mathrm{~h}^{-1}\right)$, que apresenta o menor raio iônico entre os íons lantanídeos estudados. Esta diferença pode estar relacionada a uma melhor acomodação dos ligantes PzA e dos ânions $\mathrm{Cl}^{-} \mathrm{e}$ $\mathrm{Cp}^{-}$ao redor do íon lantanídeo(III) menor, facilitando a aproximação da olefina ao sítio ativo do catalisador. Estas atividades são da mesma de ordem de grandeza das observadas por Lavini et al..$^{15}$ para $\mathrm{LnBr}_{2} \mathrm{CpPzA}, \mathrm{Ln}=\mathrm{Nd}$, Sm (4,3 e 4,6 kgPE mol Ln ${ }^{-1} \mathrm{~h}^{-1}$ bar $^{-1}$, respectivamente), por Ballard et al. ${ }^{16}$ para $\left[\mathrm{Y}\left(\eta-\mathrm{C}_{5} \mathrm{H}_{4} \mathrm{SiMe}_{3}\right)_{2} \mathrm{Me}\right]_{2}(5,1$ $\left.\mathrm{kgPE} \mathrm{mol} \mathrm{Y}^{-1} \mathrm{~h}^{-1} \mathrm{bar}^{-1}, 70^{\circ} \mathrm{C}\right)$, por Jeske et al. ${ }^{17}$ para $\left[\mathrm{Nd}\left(\mathrm{HC}_{5} \mathrm{Me}_{5}\right)_{2}\right]_{2}$ $\left(6,5 \mathrm{kgPE} \mathrm{mol} \mathrm{Nd}{ }^{-1} \mathrm{~h}^{-1}\right.$ bar $\left.^{-1}, 25^{\circ} \mathrm{C}\right)$ e por Timonen et al. ${ }^{18}$ para compostos de cloreto de ródio contendo o ligante macrocíclico $\mathrm{S}_{3}$-etano9 (6,7 kgPE mol $\mathrm{Rh}^{-1} \mathrm{~h}^{-1}$ bar $^{-1}, 10$ bar).

Os espectros na região do infravermelho dos produtos de polimerização obtidos quando se utilizou como catalisador os compostos $\mathrm{LnCl}_{2} \mathrm{Cp}(\mathrm{PzA})_{2}, \mathrm{Ln}=\mathrm{Nd}, \mathrm{Sm}$, Eu e Tb, mostraram bandas em 2919 e $2850 \mathrm{~cm}^{-1}$, atribuídas aos modos de estiramento antissimétrico e simétrico, respectivamente; bandas em 1461, 1360 e $1337 \mathrm{~cm}^{-1}$ que podem ser atribuídas aos modos de deformação de ângulo dos grupos metilenos e bandas na região $721-730 \mathrm{~cm}^{-1}$, que podem ser atribuídas aos modos "rocking" dos grupos metilenos. A banda em $730 \mathrm{~cm}^{-1}$ é atribuída a polietileno cristalino e a banda em $721 \mathrm{~cm}^{-1}$ indica a presença de regiões cristalinas e amorfas ${ }^{19}$ no polietileno obtido. Estes dados de infravermelho são apoiados pelas

Tabela 3. Dados de variação de massa (Dm) de $\mathrm{LnCl}_{2} \mathrm{Cp}(\mathrm{PzA})_{2}$

\begin{tabular}{|c|c|c|c|c|c|c|c|c|c|c|c|c|}
\hline \multirow[t]{2}{*}{ Ln } & \multicolumn{2}{|c|}{$1^{\mathrm{a}}$.etapa } & \multicolumn{2}{|c|}{$1^{\mathrm{a}}$.etapa } & \multicolumn{2}{|c|}{$2^{\mathrm{a}}$.etapa } & \multicolumn{2}{|c|}{$2^{\mathrm{a}}$.etapa } & \multicolumn{2}{|c|}{$3^{\mathrm{a}}$.etapa } & \multicolumn{2}{|c|}{$3^{\mathrm{a}}$.etapa } \\
\hline & $\%$ perda & $\%$ perda & $\operatorname{Dm}(\mathrm{mg})$ & $\mathrm{Dm}(\mathrm{mg})$ & $\%$ perda & $\%$ perda & $\operatorname{Dm}(\mathrm{mg})$ & $\mathrm{Dm}(\mathrm{mg})$ & $\%$ perda & $\%$ perda & $\operatorname{Dm}(\mathrm{mg})$ & $\mathrm{Dm}(\mathrm{mg})$ \\
\hline & calc & $\exp$ & calc & $\exp$ & calc & $\exp$ & calc & $\exp$ & calc & $\exp$ & calc & $\exp$ \\
\hline $\mathrm{Nd}$ & 23,38 & 23,65 & $-0,288$ & $-0,291$ & 23,38 & 23,59 & $-0,288$ & $-0,290$ & 15,97 & 16,07 & $-0,197$ & $-0,198$ \\
\hline $\mathrm{Sm}$ & 23,12 & 23,42 & $-0,812$ & $-0,822$ & 23,12 & 23,13 & $-0,812$ & - 0,812 & 15,87 & 15,19 & $-0,557$ & $-0,533$ \\
\hline $\mathrm{Eu}$ & 23,05 & 22,82 & $-0,878$ & $-0,869$ & 23,05 & 23,34 & $-0,878$ & $-0,889$ & 15,82 & 15,89 & $-0,603$ & $-0,606$ \\
\hline $\mathrm{Tb}$ & 22,74 & 22,68 & $-0,975$ & $-0,973$ & 22,74 & 22,72 & $-0,975$ & - 0,974 & 15,71 & 15,49 & $-0,674$ & $-0,664$ \\
\hline
\end{tabular}

Tabela 4. Espectros na região do infravermelho de $\mathrm{LnCl}_{2} \mathrm{Cp}(\mathrm{PzA})_{2}$

\begin{tabular}{|c|c|c|c|c|}
\hline $\mathrm{NdCl}_{2} \mathrm{Cp}(\mathrm{PzA})_{2}$ & $\mathrm{SmCl}_{2} \mathrm{Cp}(\mathrm{PzA})_{2}$ & $\mathrm{EuCl}_{2} \mathrm{Cp}(\mathrm{PzA})_{2}$ & $\mathrm{TbCl}_{2} \mathrm{Cp}(\mathrm{PzA})_{2}$ & atribuição \\
\hline $3412 w$ & $3415 w$ & $3419 w$ & $3422 w$ & $\mathrm{n}_{\mathrm{as}} \mathrm{NH}(\mathrm{PzA})$ \\
\hline $3264 w$ & $3271 w$ & $3268 w$ & $3271 w$ & $\mathrm{n}_{\mathrm{s}}^{\mathrm{as}} \mathrm{NH}(\mathrm{PzA})$ \\
\hline $2662 \mathrm{~m}$ & $2669 \mathrm{~m}$ & $2670 \mathrm{~m}$ & $2676 \mathrm{~m}$ & $\mathrm{n}(\mathrm{CH}) \mathrm{Cp}$ \\
\hline $1687 m-1642 m$ & $1684 m-1643 m$ & $1681 \mathrm{~m}-1646 \mathrm{~m}$ & $1677 \mathrm{~m}-1642 \mathrm{~m}$ & $\mathrm{n} \mathrm{C}=\mathrm{O}(\mathrm{PzA})$ \\
\hline $1618 \mathrm{~s}-1559 \mathrm{~m}-1520 \mathrm{~m}$ & $1616 \mathrm{~s}-1567 \mathrm{~m}-1519 \mathrm{~m}$ & $1619 \mathrm{~s}-1570 \mathrm{~m}-1520 \mathrm{~m}$ & $1616 \mathrm{~s}-1567 \mathrm{~m}-1520 \mathrm{~m}$ & vibração do anel (PzA) \\
\hline $1073 w$ & $1075 w$ & $1075 \mathrm{w}$ & $1079 w$ & $\mathrm{~g}(\mathrm{CH}) \mathrm{Cp}$ \\
\hline $1044 m$ & $1050 \mathrm{~m}$ & $1053 m$ & $1047 \mathrm{~m}$ & $\mathrm{~d}(\mathrm{CH}) \mathrm{Cp}$ \\
\hline $795 \mathrm{~m}$ & $795 m$ & $792 m$ & $794 m$ & $\mathrm{n}_{\mathrm{as}}(\mathrm{CH}) \mathrm{Cp}$ \\
\hline $667 w-615 w-$ & $663 w-615 w-$ & $663 w-615 w-$ & $663 w-615 w-$ & vibração do anel (PzA) \\
\hline $453 w-429 m$ & $451 w-432 m$ & $459 w-435 m$ & $458 w-429 m$ & \\
\hline
\end{tabular}

Os símbolos: s, m, w, significam, respectivamente, forte, média, fraca. 
medidas de DCS que mostraram $20 \%$ de produto cristalino e ponto de fusão de $126{ }^{\circ} \mathrm{C}$, quando foi utilizado como catalisador o composto $\mathrm{NdCl}_{2} \mathrm{Cp}(\mathrm{PzA})_{2}$.

\section{CONCLUSÕES}

A rota de síntese proposta neste trabalho com a obtenção dos compostos organolantanídeos a partir da reação dos compostos de coordenação com $\mathrm{NaCp}$, eliminou a etapa crítica da desidratação dos sais de partida e levou à formação de compostos do tipo $\mathrm{LnCl}_{2} \mathrm{Cp}(\mathrm{PzA})_{2}, \mathrm{Ln}$ $=\mathrm{Nd}, \mathrm{Sm}$, Eu e Tb. As curvas TG/DTG e a análise dos espectros na região do infravermelho evidenciaram uma diferença na coordenação das duas moléculas de pirazinamida aos íons lantanídeos(III). A atividade catalítica apresentada pelos compostos organolantanídeos sintetizados foi da ordem de 4,0 a 6,4 $\mathrm{kgPE} \mathrm{molLn}^{-1} \mathrm{~h}^{-1} \mathrm{bar}^{-1}$, utilizando-se a relação molar Al/Ln igual a 2000 , temperatura de $70{ }^{\circ} \mathrm{C}, 3$ bar de pressão de etileno. Estes valores de atividade são da mesma ordem de grandeza de atividades descritas em alguns trabalhos da literatura envolvendo a polimerização do etileno, onde foram utilizados como catalisadores compostos organometálicos de lantanídeos ou de metais de transição.

\section{AGRADECIMENTOS}

Os autores agradecem à Fapesp e ao CNPq pelo apoio financeiro.

\section{REFERÊNCIAS}

1. Ziegler, K.; Holzkamp, E.; Martin, H.; Breil, H.; Angew. Chem. 1955, 67, 541.

2. Sinn, H.; Kaminsky, W.; Adv. Organomet. Chem. 1980, 18, 99.

3. Britovsek, G. J. P.; Gibson, V. C.; Wass, D. F.; Angew. Chem., Int. Ed. 1999, $38,428$.

4. Gatti, P. M.; Oliveira, W.; J. Alloys Compd. 1998, 275-277, 894.

5. Vogel, A.I.; Furniss, B.S.; Vogel's Textbook of Pratical Organic Chemistry, $5^{\text {th }}$ ed, Longman: London, New York, 1989.

6. Lyle, S.J.; Rahman, Md. M.; Talanta 1963, 257.

7. Gray, A. P.; Thermochim. Acta 1970, 1, 563.

8. Miotti, R. D; Lavini, V.; Oliveira, W.; Resumos da $22^{a}$. Reunião Anual da Sociedade Brasileira de Química, Poços de Caldas, Brasil, 1999.

9. Miotti, R. D.; Tese de Doutorado, Universidade de São Paulo, Brasil, 2000.

10. Duval, C. Em Inorganic Thermogravimetric Analysis; $2^{\mathrm{a}}$ ed., Elsevier Publishing Company: Amsterdam, New York, 1963.

11. Singh, P. P.; Seth, J. N; J. Inorg. Nucl. Chem. 1975, 37, 593.

12. de Sá, G.; de Almeida, M. A. V.; J. Coord. Chem. 1980, 10, 35.

13. Allan, J. R.; Paton, A. D.; Turvey, K.; Bowley, H. J.; Gerrard, D. L.; J. Coord. Chem. 1988, 17, 255.

14. Fritz, H. P.; Adv. Organomet. Chem. 1964, 1, 239.

15. Lavini, V.; Maia, A. S.; Paulino, I. S.; Schuchardt, U.; Oliveira, W.; Inorg. Chem. Comm. 2001, 4, 582.

16. Ballard, D. G. H.; Courtis, A.; Holton, J.; McMeeking, J.; Pierce, R.; J. Chem. Soc. Chem. Comm. 1978, 994.

17. Jeske, G.; Lauke, H.; Mauermann, H.; Swepston, P. N.; Schumann, H.; Marks, T. J.; J. Am. Chem. Soc. 1985, 107, 8091.

18. Timonen, S.; Pakkanen, T. T.; J. Mol. Catal. A: Chem. 1996, 111, 267.

19. Aggarwal, S. L.; Sweeting, O. J.; Chem. Rev. 1957, 57, 665. 